ISSN 0001-6002/2001/43/1/7-10

Acta Médica Costarricense,(C2001

Colegio de Médicos y Cirujanos

\title{
Taponamiento Pericárdico
}

\author{
Kwok Ho Sánchez-Suen, ${ }^{1}$ Juan Ignacio Padilla-Cuadra ${ }^{1}$
}

\begin{abstract}
Resumen: El taponamiento pericárdico es una entidad de creciente incidencia, ${ }^{1}$ debido principalmente al incremento en los traumatismos cardiotorácicos y procedimientos invasivos. Fisiopatológicamente, se caracteriza por ser una condición en la que se limita la expansión de las cámaras cardíacas, como consecuencia de la compresión aguda de ellas por aumento del volumen y de la presión del líquido pericárdico. Este estado puede evolucionar hacia un shock obstructivo potencialmente letal si no es diagnosticado y tratado oportunamente.
\end{abstract}

Descriptores: Pericardio, taponamiento, pericardiocentesis, derrame pericárdico, fisiopatología.

Recibido: 19 de junio de 2000

Aceptado para publicación: 30 de enero de 2001

\section{Introducción}

La primera reseña de la anatomía normal del pericardio es atribuida a Hipócrates y más tarde fue ampliada por Vesalio. ${ }^{2} \mathrm{Se}$ atribuye a Galeno la primera descripción precisa del derrame pericárdico y de la constricción cardíaca. Harvey reportó en el siglo XVI el primer caso de ruptura cardíaca y hemopericardio. No fue hasta el siglo XVII que Richard Lower describió detalladamente el taponamiento pericárdico (TP), la constricción pericárdica y el pulso paradójico. Franz Schuh realizó la primera pericardiocentesis percutánea en 1840 usando un trócar. Los conceptos modernos del diagnóstico y tratamiento de las enfermedades pericárdicas están basados en los estudios de Isaacs, Beck, White, Fowler, Shabetai y Spodick. ${ }^{2-4}$

\section{Anatomía del pericardio}

El pericardio envuelve el corazón y los primeros centímetros de los grandes vasos en un saco seroso cubierto por células mesoteliales. ${ }^{3}$ El saco pericárdico normalmente contiene cerca de $50 \mathrm{ml}$ de líquido con la misma composición que el suero. ${ }^{1}$ El pericardio visceral se adosa a la superficie cardíaca formando el epicardio y posteriormente se refleja cerca de los sitios de origen de los grandes vasos para formar el pericardio parietal. La base se inserta en la porción muscular o tendinosa del hemidiafragma izquierdo. Al frente, excepto

Abreviaturas: TP, taponamiento pericárdico; PVC, presión venosa central; ECG, electrocardiograma; ECO, ecocardiograma.

${ }^{1}$ Medicina Crítica y Terapia Intensiva, Hospital Dr. Rafael Angel Calderón Guardia.

Correspondencia: Kwok Ho Sánchez Suen. Medicina Crítica y Terapia Intensiva, Hospital Dr. Rafael Angel Calderón Guardia. donde se adosa superiormente al manubrio e inferiormente al proceso xifoides por los ligamentos esterno-pericárdicos, el pericardio está separado de la pared torácica anterior por el pulmón y la pleura. Una pequeña área del pericardio parietal de tamaño variable, pero que usualmente corresponde con la mitad izquierda de la porción inferior del cuerpo del esternón y la porción medial de los cartílagos cuarto y quinto izquierdos, no está cubierta por pulmones o pleura y está en contacto directo con la pared torácica.

\section{Etiología}

De acuerdo con Spodick, ${ }^{1}$ los factores etiológicos del TP se clasifican de la siguiente manera:

1. Pericarditis idiopática

2. Pericarditis infecciosa (viral, ${ }^{5}$ bacteriana, ${ }^{6}$ tuberculosa ${ }^{7}$ )

3. Trauma: directo (trauma torácico ${ }^{8}$ o abdominal, quirúrgico, ${ }^{9}$ cateterización cardíaca, ${ }^{10}$ perforación esofágica) ó indirecto (trauma torácico no penetrante, irradiaciones terapéuticas del mediastino)

4. Neoplasias (mesotelioma, metástasis) ${ }^{11}$

5. Enfermedades de estructuras contiguas (infarto miocárdico)

6. Trastornos del metabolismo (uremia, coagulopatía, mixedema)

7. Pericarditis con derrame en vasculitis o colagenopatías (lupus eritematoso sistémico, ${ }^{12}$ fiebre reumática, artritis reumatoidea)

8. Otras causas (enfermedad del suero, síndrome de Reitter, síndrome de Loëffler, pancreatitis) 


\section{Diagnóstico}

Clínicamente se puede encontrar una disminución de la intensidad de los ruidos cardíacos o "precordio silencioso". Hay un aumento del área de matidez cardíaca. Estos signos se observan con mayor frecuencia en una fase tardía de la enfermedad. La tríada de Beck consiste de ruidos cardíacos alejados, hipotensión y distensión de las venas del cuello. Este último signo refleja el aumento de la presión venosa central (PVC) (generalmente 12-15 $\mathrm{cm} \mathrm{H}_{2} \mathrm{O}$ ) y está presente en casi todos los pacientes. ${ }^{1}$ La ausencia de la tríada no descarta el diagnóstico de TP, ya que sólo se presenta en un tercio de los pacientes. El pulso paradójico que se observa, es una variación respiratoria exagerada del pulso que disminuye la presión sistólica, la presión del pulso y la frecuencia cardíaca durante la inspiración. La radiografía de tórax rara vez es diagnóstica, pero puede revelar un ensanchamiento del mediastino o aumento de la silueta cardíaca. El electrocardiograma sirve para descartar otros trastornos cardíacos. Por lo general, se observan alteraciones inespecíficas, tales como un bajo voltaje, depresión o modificación del segmento ST sugestiva de una isquemia subepicárdica y a veces, alternancia eléctrica de las ondas P y los complejos QRS. ${ }^{13,14} \mathrm{La}$ pericardiocentesis es diagnóstica y terapéutica. Se confirma TP si se obtiene líquido pericárdico o sangre que no coagula. Una aspiración negativa no descarta el diagnóstico de TP, ya que en el $20 \%$ de las veces se obtiene punciones pericárdicas falsamente negativas, debido a que el saco pericárdico está ocupado por coágulos sanguíneos. ${ }^{15}$

El ecocardiograma es una prueba no invasiva que proporciona información valiosa y rápida. ${ }^{16,17}$ No solo permite hacer el diagnóstico sino además logra una valoración del compromiso hemodinámico sobre las cámaras cardíacas. Aunque la tomografía axial y la resonancia magnética pueden ser de utilidad diagnóstica, su uso es limitado en un paciente con TP inestable hemodinámicamente. En casos dudosos, en los cuales la estabilidad hemodinámica y el tiempo disponible lo permiten, la cateterización cardíaca y la angiografía pulmonar pueden confirmar el diagnóstico. Las pruebas de laboratorio son para descartar otras patologías, más que confirmar un TP.

Diagnóstico diferencial: El TP agudo debe ser diferenciado de otras condiciones con PVC elevada: neumotórax a tensión, insuficiencia ventricular derecha aguda, síndrome de vena cava superior, EPOC, pericarditis constrictiva, exacerbación aguda de una bronquitis crónica y embolismo pulmonar.

Fisiopatología y hemodinamia del TP: Las alteraciones fisiopatológicas producidas por un derrame pericárdico dependen principalmente de dos factores: a) el volumen del derrame y b) el curso temporal de su desarrollo. ${ }^{1}$ La elasticidad del pericardio permite la acumulación de un litro o más de líquido solo en el transcurso de semanas o meses. Incrementos agudos de fluidos tan pequeños como $200 \mathrm{ml}$, puede producir un aumento marcado en la presión intrapericárdica. Esto explica el hecho de que una pequeña remoción del fluido con la pericardiocentesis, va seguida de una dramática reducción en la presión intrapericárdica.
Figura 1

Teponamiento cardiaco por inyeccines de solución salina dentro del saco pericárdico en perro anestesiadic

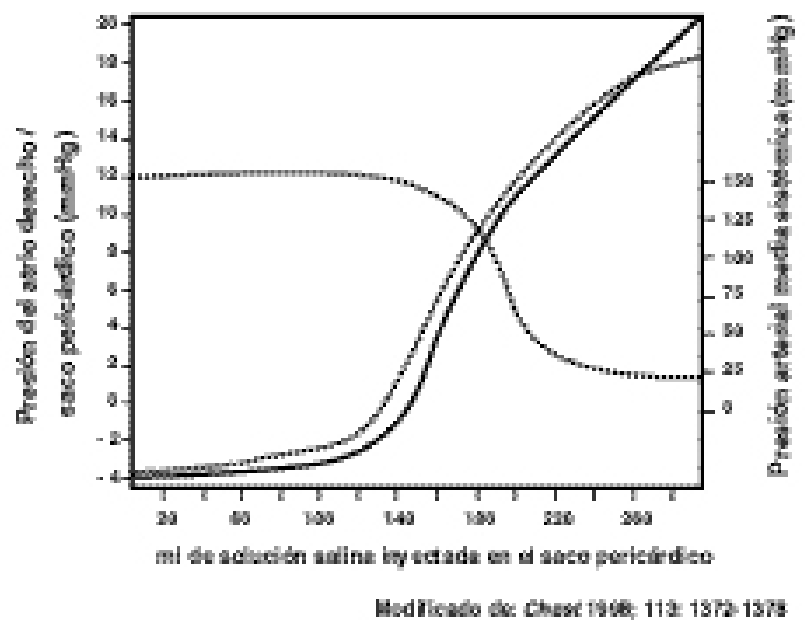

El TP produce impedimento para el llenado diastólico de las cámaras cardíacas. Conforme se alcanza la porción ascendente de la curva de presión-volumen (Figura 1), la presión intrapericárdica se incrementa y el llenado ventricular diastólico se deteriora. ${ }^{1,9}$ Conforme se incrementa la presión intrapericárdica, se impide el llenado a lo largo del ciclo cardíaco. Ante una elevación mayor de la presión en el pericardio, la PVC no puede aumentarse suficientemente para mantener el llenado diastólico y el volumen de eyección por lo tanto cae. La taquicardia refleja al principio puede mantener el gasto cardiaco, pero en vista de que se acorta más la fase de diástole, el gasto cardíaco se va reduciendo gradualmente y cae la presión arterial sistémica, apareciendo el estado de shock. En esta etapa aparece la tríada de Beck y es indicación de paro cardíaco inminente. La hipovolemia puede enmascarar las manifestaciones usuales del TP. ${ }^{18}$ Por esto, en algunos pacientes la dinámica del TP se vuelve aparente solamente luego de administración de fluidos. A pesar de que la hipervolemia usualmente acentúa las manifestaciones clínicas del $\mathrm{TP}$, la dinámica cardíaca se favorece por el incremento de la presión de llenado venticular y consecuentemente se logra sostener el gasto cardíaco. ${ }^{4,19}$

Formas de presentación: La velocidad del desarrollo y la gravedad del TP varía según el factor causal. ${ }^{1}$ En un extremo del espectro cronológico se encuentra el TP súbito que se produce como consecuencia de una ruptura cardíaca, un traumatismo penetrante, la cateterización cardíaca o la cateterización venosa central. En el otro extremo se encuentra la pericarditis con un derrame de instauración gradual, en donde los pacientes permanecen estables durante períodos prolongados; pero pueden experimentar un deterioro brusco y repentino.

\section{Tratamiento}

El problema principal consiste en resolver el derrame pericárdico hipertensivo. Una medida más inmediata puede ser el mantenimiento de cierto grado de estabilidad hemodinámica 


\section{Cuadro 1}

Alteraciones Hemodinámicas Inducidas por el Tratamiento

\begin{tabular}{lccc}
\hline Parámetro & Pericardiocentesis & Carga de volumen & Isoproterenol \\
\hline Indice sistólico $\left(\mathrm{ml}^{\prime} / \mathrm{m}^{2}\right)$ & +4 & +8 & +13 \\
Indice cardíaco $\left(\mathrm{l} / \mathrm{min} / \mathrm{m}^{2}\right)$ & $+0,0$ & $+0,7$ & $+2,3$ \\
Presión arterial media $(\mathrm{mmHg})$ & +7 & +13 & +11 \\
PVC $(\mathrm{cm} \mathrm{H}$ ) & $-2,2$ & $+5,7$ & +3 \\
Frecuencia cardíaca (lat/min) & +14 & +16 & +18 \\
Trabajo sistólico del ventrículo izquierdo $\left(\mathrm{gm} / \mathrm{m}^{2}\right)$ & +6 & +23 \\
\hline
\end{tabular}

Tomado de: Shoemaker WC (ed). Tratado de Medicina Crítica y Terapia Intensiva. Buenos Aires: Editorial Médica Panamericana, 1992:488-495.25

hasta poder corregir de forma definitiva el TP. ${ }^{20,21}$ Todos los pacientes con sospecha de un TP deben tener monitorización cardíaca continua y determinaciones de la presión sanguínea y frecuencia cardíaca cada 5-15 min. La administración de una carga líquida mejora la hemodinamia y confirma que el problema no es una insuficiencia cardíaca. ${ }^{17} \mathrm{Si}$ se produce un deterioro brusco, está indicada una pericardiocentesis de emergencia. En algunos casos (p.ej., herida penetrante al corazón, hemopericardio secundario a metástasis, etc.), la pericardiocentesis debe ir seguida de una toracotomía para la resolución definitiva. ${ }^{22}$ La pericardiocentesis mejora los parámetros hemodinámicos a través de una reducción de la presión intrapericárdica, lo que a su vez determina un aumento del retorno venoso, gasto cardíaco y perfusión coronaria (Cuadro 1). La pericardiocentesis preoperatoria reduce el riesgo de deterioro repentino con paro cardíaco durante la inducción anestésica. ${ }^{23,24}$

Pericardiocentesis: La pericardiocentesis debe ser realizada sólo por un médico entrenado en el procedimiento. Debe ser guiada por un ecocardiograma ${ }^{17,25,26}$ y se debe contar en el sitio con un equipo de resucitación cardiopulmonar al lado del paciente. El líquido que se obtiene con la punción debe ser cultivado (para bacterias, hongos y micobacterias) y analizarse por celularidad, citología, niveles de glucosa, proteínas, factor reumatoideo, anticuerpos antinucleares y niveles de complemento. ${ }^{27}$ Hay tres vías de acceso: a) subxifoidea (es la más usada porque se asocia con la menor incidencia de complicaciones), b) quinto espacio intercostal, adyacente al borde izquierdo del esternón (donde el pericardio normalmente no está cubierto por el pulmón) y c) a nivel del ápex (conlleva mayor riesgo de neumotórax y de lesionar la arteria descendente anterior). Existen riesgos significativos que acompañan al procedimiento: disritmias (incluyendo fibrilación ventricular y asistolia), punción o laceración de las cámaras cardíacas o arterias coronarias, inyección accidental de aire dentro del saco pericárdico o en cámara cardíaca, hemotórax, neumotórax y hemorragia por punción miocárdica o coronaria. ${ }^{26}$
Técnica de la pericardiocentesis por acceso subxifoideo: Se coloca el paciente en posición supina o en semi fowler a $20-30^{\circ}$. Se limpia el área alrededor del proceso xifoides con solución de yodo-povidone. Se infiltra la piel y tejidos subcutáneos con xilocaína al $2 \%$, a una profundidad de 1,5 2 pulgadas. Se localiza el sitio de punción por debajo del proceso xifoides y $1 \mathrm{~cm}$ a la izquierda. Se prensa a la aguja una pinza lagarto con la derivación V del ECG. La aguja se inserta succionando continuamente con la jeringa, a una inclinación de $45^{\circ}$ con el plano frontal y dirigiéndose hacia el hombro izquierdo. ${ }^{4,28}$ Conforme avanza, se puede sentir la resistencia que opone el pericardio. La elevación del segmento ST ocurre cuando la aguja hace contacto con la superficie ventricular (Figura 2). Si ocurre elevación del segmento PR, sugiere que ha hecho contacto con la superficie atrial. Otros signos de contacto epicárdico incluyen disritmias atriales y ventriculares, así como anormalidades de la conducción atrioventricular. La obtención de sangre o fluidos sin elevación de ST o PR o disritmias, es una indicación de que el líquido proviene del saco pericárdico más que de una cámara cardíaca. Si se obtiene sangre, no debe coagular si viene del saco pericárdico. ${ }^{29}$ Una gota del líquido sanguinolento se deja caer sobre una tela y si se esparce como una mancha rojo profundo homogéneo, debe ser sangre, pero si es del saco pericárdico, se separa en una mancha roja central y un halo periférico que es menos sanguinolento. Al final se puede insertar un catéter usando la técnica de Seldinger dentro del saco pericárdico para continuar el drenaje (Figuras 3A-3E).

\section{Figura 2}

El contacto de la aguja con el pericardio produce supradesnivel del segmento ST, que se normaliza al retirarla nuevamente

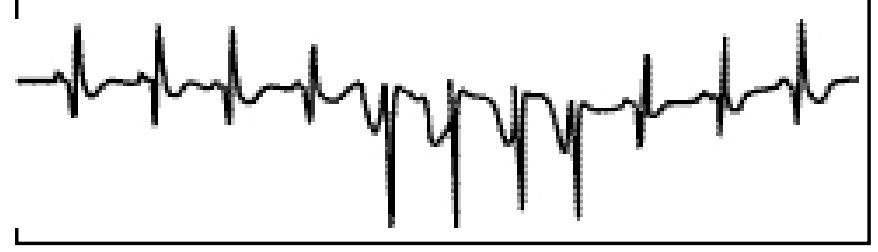




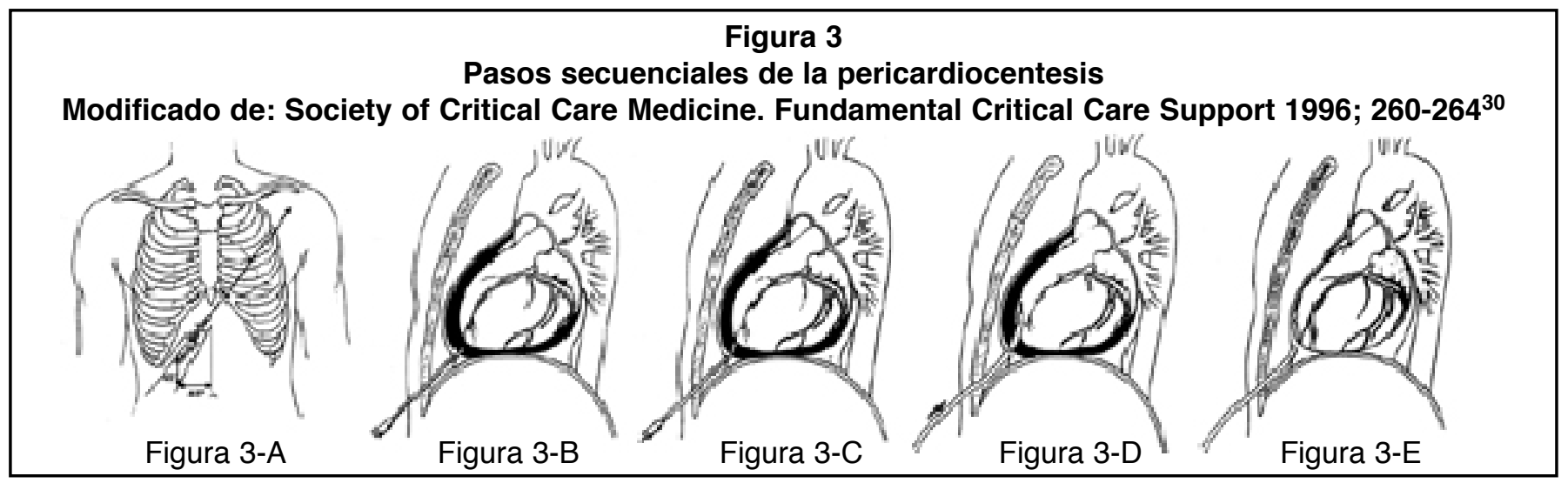

\section{Abstract}

The incidence of cardiac tamponade is increasing. This is mainly due to cardiothoracic trauma and invasive procedures. From the pathophysiological point of view, it is characterized by a restriction to the expansion of the cardiac chambers as a consequence of increased intrapericardial pressure and volume. This state can change to a obstructive shock which is potentially lethal if not promptly diagnosed and treated.

\section{Referencias}

1. Shabetai R. Function of the normal pericardium. Clin Cardiol 1999; 22 (suppl. 1): 4-5.

2. Hoit BD, Gabel M, Fowler NO. Cardiac tamponade in left ventricular dysfunction. Circulation 1990; 82:1370-1376.

3. Spodick DH. Pathophysiology of cardiac tamponade. Chest 1998; 113:1372-1378.

4. Hoit BD. Pericardial heart disease. Curr Probl Cardiol 1997; 22:357-400.

5. Silva-Cardoso J, Moura B, Martins L, Mota-Miranda A, Barr J, Donner A, et al. Pericardial involvement in human immunodeficiency virus infection. Chest 1999; 115: 418-422.

6. Snyder RW, Braun TI. Purulent pericarditis with tamponade in a postpartum patient due to group F Streptococcus. Chest 1999; 115:1746-1747.

7. Dronda F, Suzacp C. Tuberculosis pericárdica complicada con taponamiento cardíaco como forma de presentación del síndrome de inmunodeficiencia adquirida. Rev Clin Esp 1997; 197:502-506.

8. Mechem CC, Alam GA. Delayed cardiac tamponade in a patient with penetrating chest trauma. J Emerg Med 1997; 15:31-33.

9. Alam HB, Levitt A, Molyneaux R, Davidson P, Power I, Barratt S, et al. Can pleural effusions cause cardiac tamponade?. Chest 1999; 116: 1820-1822.

10. Collier PE, Blocker SH, Graff DM, Doyle P. Cardiac tamponade from central venous catheters. Am J Surg 1998; 176:212-214.

11. Pijoan P, Castro A, Centelles M, Rafel J. Taponamiento pericárdico y masa en la cavidad pericárdica como primera manifestación clínica de una neoplasia pulmonar. Rev Esp Cardiol 1998; 51:164-166.

12. Manresa JM, Gutiérrez L, Viedma P, Alfani O. Taponamiento cardíaco como presentación clínica de lupus eritematoso sistémico. Rev Esp Cardiol 1997; 50:600-602.

13. Longo MJ, Jaffe CC. Electrical alternans. N Engl J Med 1999; 341:2060.

14. Slattery DE, Dickerson DW, Pollack CV Jr. Subtle electrical alternans in a large pericardial effusion with tamponade. J Emerg Med 1997; $15: 371-372$.
15. Atar S, Chiu J, Forrester JS, Siegel RJ. Bloody pericardial effusion in patients with cardiac tamponade. Chest 1999; 116:1564-1569.

16. Tsang TS, Barnes ME, Hayes SN, Freeman WK et al. Clinical and echocardiographic characteristics of significant pericardial effusions following cardiothoracic surgery and outcomes of echo-guide pericardiocentesis for management. Mayo Clinic Experience, 1979-1998. Chest 1999; 116:322-331.

17. Tsang TS, Oh JK, Seward JB. Diagnosis and management of cardiac tamponade in the era of echocardiography. Clin Cardiol 1999; 22:446452.

18. Dwivedi SK, Saran R, Narain VS. Left ventricular diastolic collapse in low-pressure cardiac tamponade. Clin Cardiol 1998; 21:224-226.

19. Kitashiro S, Sugiura T, Tamura T, Izuoka T, McCartney JR, Boland RJ. Factors associated with increased extravascular lung water in cardiac tamponade and myocardial ischemia. Crit Care Med 1999; 27:22292233.

20. Defouilloy C, Meyer G, Slama M, Galy C, Aranda M. Intrapericardial fibrinolysis: a useful treatment in the management of purulent pericarditis. Intensive Care Med 1997; 23:117-118.

21. Fiocco M, Krasna MJ. The management of malignant pleural and pericardial effusions. Hematol Oncol Clin North Am 1997; 11:253-265.

22. Morales $\mathrm{CH}$, Salinas CM, Henao CA, Patino PA, Sorkin LS. Thoracoscopic pericardial window and penetrating cardiac trauma. J Trauma 1997; 42:273-275.

23. Salem K, Mulji A, Lonn E. Echocardiographically guided pericardiocentesis: the gold standard for the management of percardial effusion and cardiac tamponade. Can J Cardiol 1999; 15:1251-1255.

24. Larose E, Ducharme A, Mercier LA, Pelletier G, Krauss B, Greeen SM, McCartney JR, Boland RJ. Prolonged distress and clinical deterioration before pericardial drainage in patients with cardiac tamponade. Can $\mathbf{J}$ Cardiol 2000; 16:331-336.

25. Shoemaker WC. Taponamiento pericárdico. En: Shoemaker WC (ed). Tratado de Medicina Crítica y Terapia Intensiva. Buenos Aires: Editorial Médica Panamericana, 1992:488-495.

26. Fagan SM, Chan KL. Pericardiocentesis: blind no more!. Chest 1999; 116:322-331.

27. Mueller XM, Tevaearai HT, Hurni M, Ruchat $P$ et al. Etiologic diagnosis of pericardial disease: the value of routine tests during surgical procedures. J Am Coll Surg 1997; 184:645-649.

28. McAllister HA, Hall RJ, Cooley DA. Tumors of the heart and percardium. Curr Probl Cardiol 1999; 24:61-116.

29. Merce J, Sagrista J, Permanyer G, Soler J. Should pericardial drainage be performed routinely in patients who have a large pericardial effusion without tamponade?. Am J Med 1998; 105:106-109.

30. Society of Critical Care Medicine. Fundamental Critical Care Support 1996; 260-264. 